\title{
Tyre induced vibrations of the car-trailer system
}

\author{
S. Beregi ${ }^{\mathrm{a}}$, D. Takács ${ }^{\mathrm{b}}$, G. Stépán ${ }^{\mathrm{a}}$ \\ ${ }^{a}$ Department of Applied Mechanics, Budapest University of Technology and Economics \\ ${ }^{b}$ MTA-BME Research Group on Dynamics of Machines and Vehicles
}

\begin{abstract}
The lateral and yaw dynamics of the car-trailer combination are analysed by means of a single track model. The equations of motion are derived rigorously by means of the Appell-Gibbs equations for constant longitudinal velocity of the vehicle. The tyres are described with the help of the so-called delayed tyre model, which is based on a brush model with pure rolling contact. The lateral forces and aligning torques of the tyre/road interaction are calculated via the instantaneous lateral deformations in the contact patches. The linear stability analysis of the rectilinear motion is performed via the analytically determined characteristic function of the system. Stability charts are constructed with respect to the vehicle longitudinal velocity and the payload position on the trailer. Self-excited lateral vibrations are detected with different vibration modes at low and at high longitudinal speeds of the vehicle. The effects of the tyre parameters are also investigated.
\end{abstract}

Keywords: Tyre/road interaction, Car-trailer lateral stability, Self-excited vibrations

\section{Introduction}

The tiny contact regions between tyres and road surface have essential effects on the dynamics of large-scale road vehicles. Consequently, tyre deformations strongly influence vehicle manoeuvrability and stability, which explains the fact that tyre dynamics is one of the most important research fields in vehicle dynamics.

Several tyre models have been developed to be used in analytical and/or numerical investigations of vehicle systems. The basic models assume quasi-static tyre deformations both inside and outside the tyre-ground contact patch. The tyre model initially developed in [1] is based on the so-called creep-force idea that has become standard in vehicle dynamics. In engineering applications, this model can efficiently be used for different vehicle set-ups running with large or medium speeds. The usability of this tyre model has been proven by many studies; here, we mention recent work on aircraft ground dynamics only (see, for example, $[2,3,4]$ ). Nevertheless, more accurate models are needed when the instantaneous shape of the deformed tyre is relevant. The corresponding dynamic tyre models that can be used in multi-body simulations of complex vehicle systems are reviewed and discussed in [5].

During the last decades, the fuel consumption and noise generation of vehicles have become relevant aspects of tyre development. Recent publications, like $[6,7,8,9]$, observe that the tyre-ground interaction has a key role in noise generation and consequently, it has a certain effect on fuel consumption. These publications use tyre-ground contact models in order to take into account the excitation caused by the rough road surface, so they explain noise by forced vibrations. With a similar mechanism, self-excited vibrations may also contribute to the noise generation. Also, the interaction between the tyre contact patch and the related vehicle system can lead to small amplitude selfexcited vibrations, which can also cause increased level of heat and noise. In [10], a simple mechanical model of a shimmying tyre is analysed, and the existence of such critical parameter domains are experimentally validated where small amplitude self-excited vibrations cause the increase of tyre temperature and rolling resistance.

The further development of dynamic tyre-ground contact models are also required by the driving aid systems, especially the self-parking systems, which tend to become increasingly common nowadays. These systems demand accurate modelling of the vehicle motion by means of improved tyre models. While the classical quasi-stationary

Email addresses: sandor .beregi@gmail.com (S. Beregi), takacs@mm. bme.hu (D. Takács), stepan@mm.bme.hu (G. Stépán) 
tyre models can be improved by introducing more and more characteristic parameters obtained from extensive experimental work, there are several arguments for not to over-complicate these models. On the one hand, the real-time simulation of the vehicle models and the real-time digital control of the corresponding vehicles require the use of simple-enough dynamic tyre models. On the other hand, analytical studies are also needed to find new insights into the parameter dependence of vehicle dynamic behaviour. These investigations prefer the use of complex-enough tyre models but with low number of parameters. As a typical example for such approach, the single track model of a passenger car ('bicycle model') can be mentioned in [11], where the non-linear analysis of the turning manoeuvre is carried out leading to new information about the lateral stability of vehicles.

In the present report, the stability of the car-trailer combination is studied. Although this vehicle system was thoroughly analysed in former studies $[12,13,14]$, it is worth revisiting it with the use of an improved dynamic tyre model, since the bicycle model of the four-wheeled car in [15] showed that new unstable parameter domains can be found by using a time delayed tyre model (also called 'tyre with memory effect'). These results motivated us to focus on the calculation of the instantaneous shape of the tyre-ground contact patch center-line and to implement the time delayed tyre model in a car-trailer system, which is capable to describe the so-called regenerative vibrations of the tyres, too.

It is shown in [16] for a shimmying wheel that the omission of the tyre deformation outside the contact patch (also called tyre relaxation) does not influence the linear stability charts qualitatively, namely, the stability charts have the same structure in case of zero and non-zero tyre relaxation. Experiments in [17] also show that the brush tyre model considering the lateral deformation of the tyre only can be efficiently used for the investigation of the lateral stability of the towed tyre. Based on this, in order to keep the governing equations analytically manageable, the partial slip in the tyre/ground contact as well as the tyre deformation outside the contact patch are neglected. These are rough but acceptable approximations in case of the linear stability analysis of the stationary rectilinear motion when small tyre deformations are assumed. As a result of this analysis, stability charts are constructed with respect to the speed of the vehicle and the location of the trailer's payload. The effects of the tyre damping and the parameters of the trailer's tyre are investigated in details.

\section{Mechanical model}

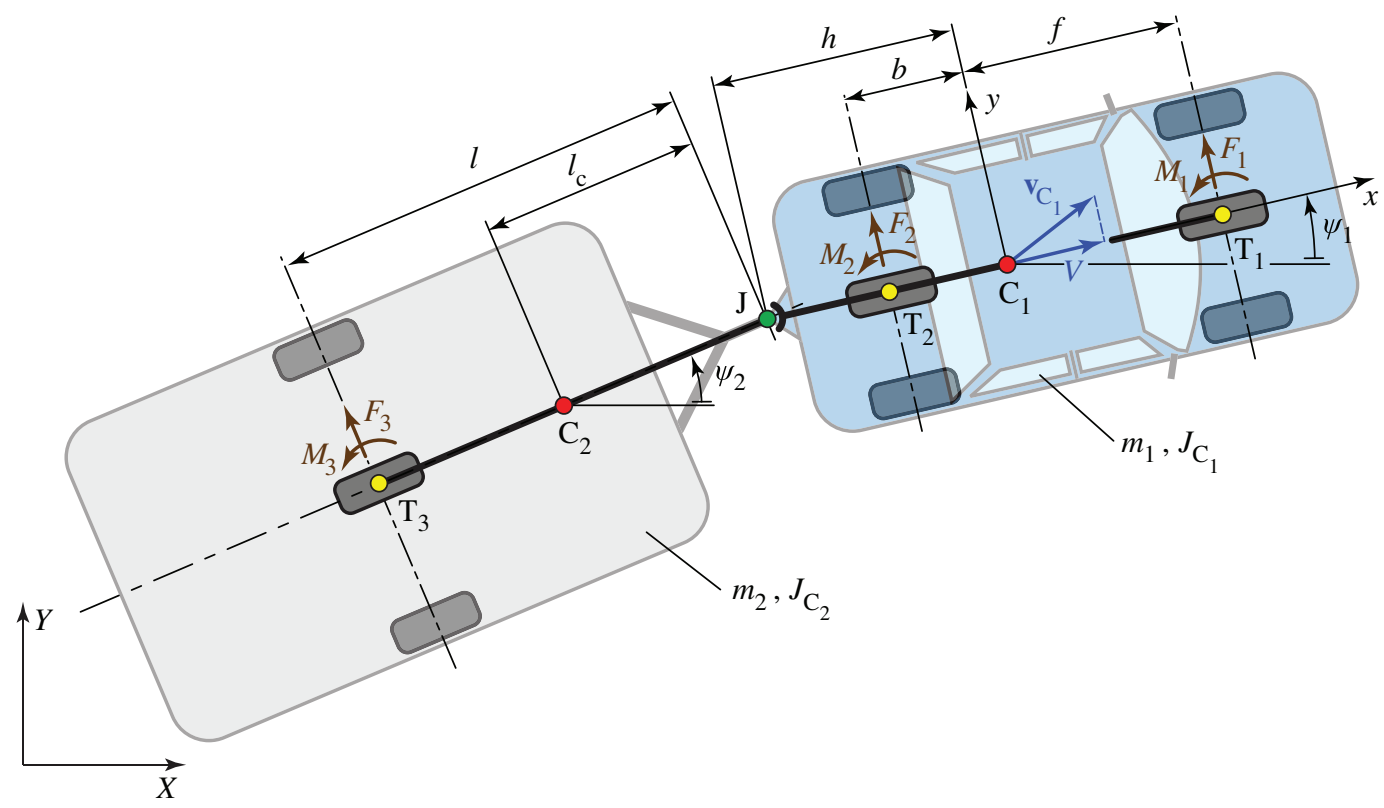

Figure 1: The single track model of the car-trailer combination.

The mechanical model is shown in Fig. 1. The car-trailer combination is represented by the so-called single track 
model (in-plane bicycle model with a trailer, see [12]) by means of the following assumptions. The vehicle can move in the $(X, Y)$ plane, and its motion is described in the ground-fixed Lagrangian coordinate system. The vertical motion of the vehicle is neglected. There are several mechanisms how the vertical dynamics can influence lateral stability. On the one hand, the vertical load at the hitch point has an effect on the friction forces at the hitch. The variation of the hitch point load modifies the friction torque that dissipates the energy of the oscillations. Not considering this damping effect is a conservative estimation from stability viewpoint. On the other hand, the hitch point load has an effect on the load of the car rear axle, too, which influences the sizes of the contact patches and the corresponding lateral stiffness of the tyres. These effects can be modelled by means of different tyre parameters in the mechanical model. By using the single track model, we also neglect the lateral extension of the vehicles, which is an acceptable simplification for the stability analysis of stationary rectilinear motion.

Hence, the vehicle system is represented by two rods, which are connected with a rotational joint at J. The wheels are attached to the vehicle at points $\mathrm{T}_{1}, \mathrm{~T}_{2}$ and $\mathrm{T}_{3}$. The notations $h, f$ and $b$ stand for the distances between the centre of mass $\mathrm{C}_{1}$ and the joint $\mathrm{J}$, the front and rear wheel point $\mathrm{T}_{1}$ and $\mathrm{T}_{2}$ respectively. The distance between the centre of gravity $\mathrm{C}_{2}$ of the trailer and the king pin $\mathrm{J}$ is referred to as $l_{\mathrm{c}}$, while $l$ (often called caster/trail length) is the distance between the king pin $\mathrm{J}$ and the wheel axle of the trailer. Four generalized coordinates describe the motion of the vehicle: $X_{1}$ and $Y_{1}$, which are the coordinates of the centre of gravity $\mathrm{C}_{1}$, and the angles $\psi_{1}$ and $\psi_{2}$ of the longitudinal axes of the car and the trailer with respect to the $X$ direction. These are represented in the vector of generalized coordinates:

$$
\mathbf{q}=\left[\begin{array}{llll}
X_{1} & Y_{1} & \psi_{1} & \psi_{2}
\end{array}\right]^{T}
$$

One kinematic constraint is applied to the system, namely, the longitudinal velocity $V$ of the car is kept constant in time:

$$
\dot{X}_{1} \cos \psi_{1}+\dot{Y}_{1} \sin \psi_{1}=V
$$

where dots refer to the derivatives with respect to time. The equations of motion of the non-holonomic mechanical system can be determined efficiently by means of the Appell-Gibbs equation [18]. This requires the definition of the so-called pseudo velocities $\beta_{1,2,3}$, which can be introduced with the help of a system of linear equations:

$$
\left[\begin{array}{cccc}
\cos \psi_{1} & \sin \psi_{1} & 0 & 0 \\
-\sin \psi_{1} & \cos \psi_{1} & 0 & 0 \\
0 & 0 & 1 & 0 \\
0 & 0 & 0 & 1
\end{array}\right]\left[\begin{array}{c}
\dot{X}_{1} \\
\dot{Y}_{1} \\
\dot{\psi}_{1} \\
\dot{\psi}_{2}
\end{array}\right]=\left[\begin{array}{c}
V \\
\beta_{1} \\
\beta_{2} \\
\beta_{3}
\end{array}\right]
$$

where the coefficient matrix provides the unique relationship between the pseudo velocities $\beta_{r}$ and the generalized velocities $\dot{q}_{k}$. The first row of this matrix is given by the kinematic constraint Eq. (2), whereas the other rows can be chosen intuitively. Here, $\beta_{1}$ is the lateral velocity of centre of gravity $\mathrm{C}_{1}$ of the car, while $\beta_{2}$ and $\beta_{3}$ are the angular velocities of the car and the trailer, respectively.

By means of Eq. (3), the generalized velocities can be eliminated using the pseudo velocities, which already fulfil the kinematic constraint:

$$
\begin{aligned}
& \dot{X}_{1}=V \cos \psi_{1}-\beta_{1} \sin \psi_{1}, \\
& \dot{Y}_{1}=V \sin \psi_{1}+\beta_{1} \cos \psi_{1}, \\
& \dot{\psi}_{1}=\beta_{2}, \\
& \dot{\psi}_{2}=\beta_{3} .
\end{aligned}
$$

The energy of acceleration containing the pseudo accelerations can be calculated from:

$$
S=\frac{1}{2} m_{1} a_{\mathrm{C}_{1}}^{2}+\frac{1}{2} J_{\mathrm{C}_{1}} \varepsilon_{1}^{2}+\frac{1}{2} m_{2} a_{\mathrm{C}_{2}}^{2}+\frac{1}{2} J_{\mathrm{C}_{2}} \varepsilon_{2}^{2}+\ldots
$$

where $m_{1}$ and $m_{2}$ are the masses, $J_{\mathrm{C}_{1}}$ and $J_{\mathrm{C}_{2}}$ are the mass moments of inertia (yaw inertias) about the $Z$ axis at the centres of gravity of the car and the trailer, respectively, $a_{\mathrm{C}_{1}}$ and $a_{\mathrm{C}_{2}}$ are the accelerations of the centres of gravity, 
whereas $\varepsilon_{1}$ and $\varepsilon_{2}$ are the angular accelerations. All these kinematic quantities can be given as a function of the pseudo velocities $\left(\beta_{1}, \beta_{2}, \beta_{3}\right)$ and pseudo accelerations $\left(\dot{\beta}_{1}, \dot{\beta}_{2}, \dot{\beta}_{3}\right)$, and the energy of acceleration can be expressed as

$$
\begin{aligned}
S= & \frac{1}{2}\left(\left(m_{1}+m_{2}\right) \dot{\beta}_{1}^{2}+\left(J_{\mathrm{C}_{1}}+m_{2} h^{2}\right) \dot{\beta}_{2}^{2}+\left(J_{\mathrm{C}_{2}}+m_{2} l_{\mathrm{c}}^{2}\right) \dot{\beta}_{3}^{2}\right) \\
& -m_{2} h \dot{\beta}_{1} \dot{\beta}_{2}-m_{2} l_{\mathrm{c}}\left(\dot{\beta}_{1}-h \dot{\beta}_{2}\right) \dot{\beta}_{3} \cos \left(\psi_{2}-\psi_{1}\right) \\
& +\left(\left(m_{1}+m_{2}\right) V \beta_{2}+m_{2} l_{\mathrm{c}} \beta_{3}^{2} \sin \left(\psi_{2}-\psi_{1}\right)\right) \dot{\beta}_{1} \\
& -m_{2} h\left(V \beta_{2}+l_{\mathrm{c}} \beta_{3}^{2} \sin \left(\psi_{2}-\psi_{1}\right)\right) \dot{\beta}_{2} \\
& -m_{2} l_{\mathrm{c}}\left(V \cos \left(\psi_{2}-\psi_{1}\right)+\left(\beta_{1}-h \beta_{2}\right) \sin \left(\psi_{2}-\psi_{1}\right)\right) \beta_{2} \dot{\beta}_{3}+\ldots
\end{aligned}
$$

The presented formula is not complete, but it can be seen, that only the given terms are necessary for the derivation of the governing equations.

The Appell-Gibbs equations read

$$
\frac{\partial S}{\partial \dot{\beta}_{r}}=\Gamma_{r}, \quad r=1,2,3,
$$

where $\Gamma_{r}$ are the pseudo forces, which can be identified as the coefficients of the virtual pseudo velocities in the virtual power of the active forces:

$$
\delta P=\sum_{r=1}^{3} \Gamma_{r} \delta \beta_{r}
$$

In our case, the virtual power can be calculated via the formula

$$
\delta P=\mathbf{F}_{1} \cdot \delta \mathbf{v}_{\mathrm{T}_{1}}+\mathbf{F}_{2} \cdot \delta \mathbf{v}_{\mathrm{T}_{2}}+\mathbf{F}_{3} \cdot \delta \mathbf{v}_{\mathrm{T}_{3}}+\left(\mathbf{M}_{1}+\mathbf{M}_{2}\right) \cdot \delta \omega_{1}+\mathbf{M}_{3} \cdot \delta \omega_{2},
$$

where $\mathbf{F}_{i}$ and $\mathbf{M}_{i}$ (for $i=1,2,3$ ) are the forces and aligning torques transmitted by the tyres from the ground to the vehicle. They can be written in the car-fixed $(x, y, z)$ coordinate system (see Fig. 1) as

$$
\mathbf{F}_{1}=\left[\begin{array}{c}
0 \\
F_{1} \\
0
\end{array}\right], \quad \mathbf{F}_{2}=\left[\begin{array}{c}
0 \\
F_{2} \\
0
\end{array}\right], \quad \mathbf{F}_{3}=\left[\begin{array}{c}
-F_{3} \sin \left(\psi_{2}-\psi_{1}\right) \\
F_{3} \cos \left(\psi_{2}-\psi_{1}\right) \\
0
\end{array}\right],
$$

and $\mathbf{M}_{i}=M_{i} \mathbf{k}$, where $\mathbf{k}$ is the unit vector of the $Z$ direction. The notations $\delta \mathbf{v}_{\mathrm{T}_{1}}, \delta \mathbf{v}_{\mathrm{T}_{2}}$ and $\delta \mathbf{v}_{\mathrm{T}_{3}}$ are the virtual velocities of points $\mathrm{T}_{1}, \mathrm{~T}_{2}$ and $\mathrm{T}_{3}$, respectively, whereas $\delta \omega_{1}=\delta \beta_{2} \mathbf{k}$ and $\delta \omega_{2}=\delta \beta_{3} \mathbf{k}$ are the virtual angular velocities. The virtual velocities can easily be calculated in the car-fixed $(x, y, z)$ coordinate system:

$$
\begin{aligned}
& \delta \mathbf{v}_{\mathrm{T}_{1}}=\delta\left[\begin{array}{c}
V \\
\beta_{1}+f \beta_{2} \\
0
\end{array}\right], \quad \delta \mathbf{v}_{\mathrm{T}_{2}}=\delta\left[\begin{array}{c}
V \\
\beta_{1}-b \beta_{2} \\
0
\end{array}\right], \\
& \delta \mathbf{v}_{\mathrm{T}_{3}}=\delta\left[\begin{array}{c}
V+l \beta_{3} \sin \left(\psi_{2}-\psi_{1}\right) \\
\beta_{1}-h \beta_{2}-l \beta_{3} \cos \left(\psi_{2}-\psi_{1}\right) \\
0
\end{array}\right] .
\end{aligned}
$$

After some manipulation of the given formulas, one can determine both sides of Eq. (7), and the governing equations of the non-linear system can be composed as

$$
\begin{aligned}
& m_{1}\left(\dot{\beta}_{1}+V \beta_{2}\right)+m_{2}\left(\dot{\beta}_{1}+V \beta_{2}-h \dot{\beta}_{2}-l_{\mathrm{c}} \dot{\beta}_{3} \cos \left(\psi_{2}-\psi_{1}\right)+l_{\mathrm{c}} \beta_{3}^{2} \sin \left(\psi_{2}-\psi_{1}\right)\right) \\
& \quad=F_{1}+F_{2}+F_{3} \cos \left(\psi_{2}-\psi_{1}\right) \\
& J_{\mathrm{C}_{1}} \dot{\beta}_{2}+m_{2}\left(h^{2} \dot{\beta}_{2}-h V \beta_{2}-h \dot{\beta}_{1}+h l_{\mathrm{c}} \dot{\beta}_{3} \cos \left(\psi_{2}-\psi_{1}\right)-h l_{\mathrm{c}} \beta_{3}^{2} \sin \left(\psi_{2}-\psi_{1}\right)\right) \\
& \quad=F_{1} f-F_{2} b-F_{3} h \cos \left(\psi_{2}-\psi_{1}\right)+M_{1}+M_{2}, \\
& J_{\mathrm{C}_{2}} \dot{\beta}_{3}+m\left(l_{\mathrm{c}}^{2} \dot{\beta}_{3}-l_{\mathrm{c}} V \beta_{2} \cos \left(\psi_{2}-\psi_{1}\right)-l_{\mathrm{c}} \dot{\beta}_{1} \cos \left(\psi_{2}-\psi_{1}\right)-l_{\mathrm{c}} \beta_{1} \beta_{2} \sin \left(\psi_{2}-\psi_{1}\right)\right. \\
& \left.\quad+h l_{\mathrm{c}} \dot{\beta}_{2} \cos \left(\psi_{2}-\psi_{1}\right)+h l_{\mathrm{c}} \beta_{2}^{2} \sin \left(\psi_{2}-\psi_{1}\right)\right)=M_{3}-F_{3} l .
\end{aligned}
$$


The linearised form of these equations of motion is already given in [12]. We present here the equations with all the geometric non-linearities, which can be required for the non-linear analyses of the model, that will be carried out in future work. Our model does not consider the vertical dynamics of the vehicle, which strongly limited its applicability in case of manoeuvres when the vertical forces on tyres suddenly change. Nevertheless, single track models are often used to investigate the stability of different manoeuvres (see, for example, [11]).

\section{Tyre model with memory effect}

In order to calculate the forces and aligning torques of the tyres, a delayed tyre model is used, which is based on the so-called brush tyre model (see [12]). Let us consider the tyre of a rigid wheel with the wheel centre point $\mathrm{T}$. The wheel centre point position is described by the $X_{\mathrm{T}}$ and $Y_{\mathrm{T}}$ coordinates while the angle of the wheel plane is denoted by $\psi$. The tyre-ground contact patch with its laterally deformed centre line is shown in Fig. 2. The length of the contact patch is $2 a$. The tyre is modelled as massless elements with distributed stiffness $k$ and damping $d$, and the lateral displacement of its centre line is described by the deformation function $q(x, t)$. The wheel rotates around the $y$ axis and due to this rotation, the tyre particles in the contact patch move in the wheel-fixed $(x, y, z)$ Eulerian coordinate system, although they have zero velocity relative to the ground (in case of rolling). Since the $z$ coordinate of a contacted tyre particle is constant, the relative motion of the tyre particles in the contact patch can be investigated as an in-plane motion. Thus, we omit the $z$ coordinate in our further calculations in order to keep the formulas as simple as possible.

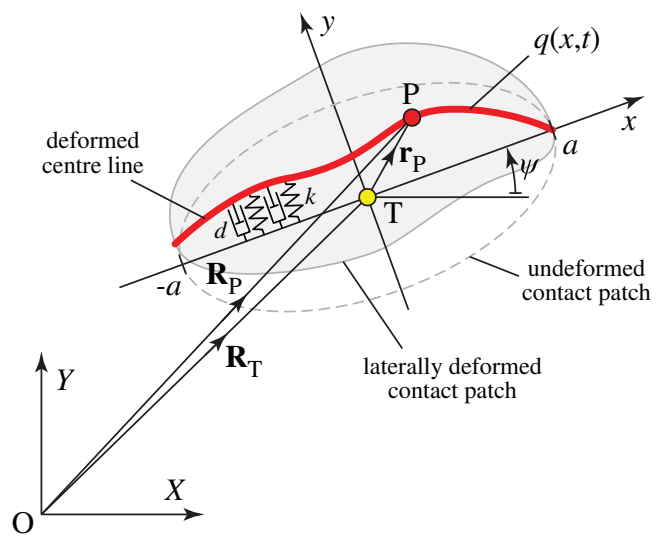

Figure 2: Tyre deformation in the contact patch.

The position vector $\mathbf{R}_{\mathrm{P}}$ of an arbitrary point $\mathrm{P}$ on the contact patch centre line can be described with the help of the wheel centre point $\mathrm{T}$ (i.e. the origin of the $(x, y, z)$ Eulerian coordinate system):

$$
\mathbf{R}_{\mathrm{P}}=\mathbf{R}_{\mathrm{T}}+\mathbf{R}_{\mathrm{TP}}
$$

where the position vectors $\mathbf{R}_{\mathrm{P}}, \mathbf{R}_{\mathrm{T}}$ and $\mathbf{R}_{\mathrm{TP}}$ are given in the $(X, Y, Z)$ ground-fixed coordinate system. Give the position of the wheel centre point by

$$
\mathbf{R}_{\mathrm{T}}=\left[\begin{array}{c}
X_{\mathrm{T}} \\
Y_{\mathrm{T}}
\end{array}\right]
$$

The position vector of the point $\mathrm{P}$ is given in the $(x, y, z)$ coordinate system by

$$
\mathbf{r}_{\mathrm{P}}=\left[\begin{array}{c}
x \\
q(x, t)
\end{array}\right] \text {. }
$$

This can be also expressed in the ground-fixed coordinate system:

$$
\mathbf{R}_{\mathrm{TP}}=\left[\begin{array}{l}
x \cos \psi-q(x, t) \sin \psi \\
x \sin \psi+q(x, t) \cos \psi
\end{array}\right] .
$$


Hence, the the position vector $\mathbf{R}_{\mathrm{P}}$ of an arbitrary contact point reads as

$$
\mathbf{R}_{\mathrm{P}}=\left[\begin{array}{c}
X_{\mathrm{T}}+x \cos \psi-q(x, t) \sin \psi \\
Y_{\mathrm{T}}+x \sin \psi+q(x, t) \cos \psi
\end{array}\right] .
$$

By assuming pure rolling, the velocity of the points in the contact patch relative to the ground is zero, namely:

$$
\frac{\mathrm{d}}{\mathrm{d} t} \mathbf{R}_{\mathrm{P}}=\mathbf{0} .
$$

Due to the material flow along the $x$ direction via the rotation of the wheel, this leads to:

$$
\begin{aligned}
& \dot{X}_{\mathrm{T}}+v \cos \psi-x \dot{\psi} \sin \psi-\frac{\mathrm{d}}{\mathrm{d} t} q(x, t) \sin \psi-q(x, t) \dot{\psi} \cos \psi=0, \\
& \dot{Y}_{\mathrm{T}}+v \sin \psi+x \dot{\psi} \cos \psi+\frac{\mathrm{d}}{\mathrm{d} t} q(x, t) \cos \psi-q(x, t) \dot{\psi} \sin \psi=0,
\end{aligned}
$$

in which $v$ is the longitudinal component of the velocity-field (i.e. the longitudinal translational rate of the tyre particles) in the Eulerian system. The material time derivative of $q(x, t)$ is:

$$
\frac{\mathrm{d}}{\mathrm{d} t} q(x, t)=\dot{q}(x, t)+q^{\prime}(x, t) v
$$

where prime refers to the derivative with respect to the space coordinate $x$. After multiplying Eq. (19) with $\sin \psi$, and similarly Eq. (20) with $\cos \psi$, and adding the two equations together the following can be written:

$$
\frac{\mathrm{d}}{\mathrm{d} t} q(x, t)=\dot{X}_{\mathrm{T}} \sin \psi-\dot{Y}_{\mathrm{T}} \cos \psi-x \dot{\psi}
$$

And in the same way, multiplying Eq. (19) with $\cos \psi$, and Eq. (20) with $\sin \psi$ and adding them together, we obtain:

$$
v=-\dot{X}_{\mathrm{T}} \cos \psi-\dot{Y}_{\mathrm{T}} \sin \psi+q(x, t) \dot{\psi}
$$

Substituting Eq. (22) and Eq. (23) into Eq. (21) a non-linear partial differential equation (PDE) can be obtained:

$$
\dot{q}(x, t)=\dot{X}_{\mathrm{T}} \sin \psi-\dot{Y}_{\mathrm{T}} \cos \psi-x \dot{\psi}+q^{\prime}(x, t)\left(\dot{X}_{\mathrm{T}} \cos \psi+\dot{Y}_{\mathrm{T}} \sin \psi-\dot{\psi} q(x, t)\right)
$$

for $x \in[-a, a]$. This equation can be originated in the kinematic constraint Eq. (18) of rolling, and within the limits of our model it describes the instantaneous shape of the contact patch center-line in case of pure rolling.

The boundary condition (BC) of the PDE is $q(a, t)=0$, which corresponds to the zero deformation at the leading edge according to the implemented brush tyre model.

The tyre forces and aligning torques caused by tyre deformation can be calculated by the integral formulas:

$$
\begin{aligned}
& F=k \int_{-a}^{a} q(x, t) \mathrm{d} x+d \int_{-a}^{a} \frac{\mathrm{d}}{\mathrm{d} t} q(x, t) \mathrm{d} x, \\
& M=k \int_{-a}^{a} x q(x, t) \mathrm{d} x+d \int_{-a}^{a} x \frac{\mathrm{d}}{\mathrm{d} t} q(x, t) \mathrm{d} x .
\end{aligned}
$$

One can use the formulas of Eq. (25) together with Eq. (24) for each tyre of the mechanical model in Fig. 1. And then, Eq. (12) turns to be three non-linear integro-differential equations (IDEs) coupled to three non-linear PDEs.

In what follows, it is shown that the IDE-PDE system can be transformed into a more manageable form. We can compose the travelling wave solution of the PDEs and we can obtain delay differential equations (DDEs) as governing equations. This method provides simpler system of equations with respect to three scalar time-dependent coordinates only, while the lateral deformation shape of the tyre is still described accurately (for rolling), while the governing equations still represent the infinite dimensional behaviour of the original system. 


\subsection{The travelling wave solution for rolling tyres}

In order to find the travelling wave solution of the PDE given in Eq. (24), the position vector $\mathbf{R}_{\mathrm{P}}$ of an arbitrary contact point has to be used. Since tyre particles keep their positions in the ground-fixed coordinate system during the contact, we can construct the travelling wave solution as

$$
\mathbf{R}_{\mathrm{P}}(x, t)=\mathbf{R}_{\mathrm{P}}(a, t-\tau(x)),
$$

where $\tau(x)$ is the time delay, which is the function of the coordinate $x$. This time delay is needed for a tyre particle to travel from the leading edge $(x=a)$ to its actual position characterized by $x$. With the help of Eq. (17) in Eq. (26), the travelling wave solution can be composed, which satisfy the PDE Eq. (24) with boundary condition $q(a, t)=0$ :

$$
\begin{aligned}
q(x, t) & =\left(X_{\mathrm{T}}(t)-X_{\mathrm{T}}(t-\tau)\right) \sin \psi(t)-\left(Y_{\mathrm{T}}(t)-Y_{\mathrm{T}}(t-\tau)\right) \cos \psi(t) \\
& -a \sin (\psi(t)-\psi(t-\tau)),
\end{aligned}
$$

for $x \in[-a, a]$. All this means that the lateral deformation of a tyre can be calculated via the past and present values of the wheel centre point coordinates $\left(X_{\mathrm{T}}, Y_{\mathrm{T}}\right)$ and the wheel deflection angle $(\psi)$.

Unfortunately, the relationship between the space coordinate $x$ and the time delay $\tau$ reads:

$$
\begin{aligned}
x & =-\left(X_{\mathrm{T}}(t)-X_{\mathrm{T}}(t-\tau)\right) \cos \psi(t)+\left(Y_{\mathrm{T}}(t)-Y_{\mathrm{T}}(t-\tau)\right) \sin \psi(t) \\
& +a \cos (\psi(t)-\psi(t-\tau)),
\end{aligned}
$$

namely, the time delay can not be expressed explicitly. However, we can calculate the derivative of the coordinate $x$ with respect to the time delay

$$
\begin{aligned}
\frac{\partial x}{\partial \tau} & =-\dot{X}_{\mathrm{T}}(t-\tau) \cos \psi(t)+\dot{Y}_{\mathrm{T}}(t-\tau) \sin \psi(t) \\
& -a \sin (\psi(t)-\psi(t-\tau)) \dot{\psi}(t-\tau) .
\end{aligned}
$$

In Eq. (25), one can use these expressions to change the integration variable $x$ to $\tau$.

\subsection{Tyre force and aligning torque for small oscillations around the straight wheel motion}

As it was mentioned before, the equation of motion of the car-trailer system can be transformed into DDEs. In order to do this, the travelling wave solution Eq. (27) with Eq. (28) and Eq. (29) can be used in Eq. (25) for each of the three tyres. Here we show this calculation only for the linear case since we focus on the linear stability analysis of the car-trailer system.

Thus, we can linearise the equations around the rectilinear forward motion of the tyre, which corresponds to

$$
\begin{array}{lll}
X_{\mathrm{T}}(t)=V t, & Y_{\mathrm{T}}(t) \equiv 0, & \psi(t) \equiv 0, \\
\dot{X}_{\mathrm{T}}(t) \equiv V, & \dot{Y}_{\mathrm{T}}(t) \equiv 0, & \dot{\psi}(t) \equiv 0 .
\end{array}
$$

For the small oscillations around this solution, the linearised form of the travelling wave solution (27) can be used:

$$
q(x, t)=V \tau \psi(t)-\left(Y_{\mathrm{T}}(t)-Y_{\mathrm{T}}(t-\tau)\right)-a(\psi(t)-\psi(t-\tau)) .
$$

For this linear case, the relationship (28) between the space coordinate $x$ and the time delay $\tau$ simplifies to:

$$
x=a-V \tau \Rightarrow \tau=\frac{a-x}{V},
$$

whereas the velocity field given in Eq. (23) reduces to

$$
v=-V .
$$

Considering these, the material time derivative in Eq. (22) of the lateral tyre deformation gives:

$$
\frac{\mathrm{d}}{\mathrm{dt}} q(x, t)=(V \tau-a) \dot{\psi}(t)-\dot{Y}_{\mathrm{T}}(t)+V \psi(t) .
$$


The forces and aligning torques caused by tyre deformation can be calculated with the help of Eq. (25), where the integrals can be transformed by replacing the space coordinate $x$ to the time delay $\tau$. According to the formulas in Eq. (32), we can use:

$$
\mathrm{d} x=-V \mathrm{~d} \tau
$$

whereas the limits of the integrations get modified to

$$
\begin{aligned}
& x=-a \quad \Rightarrow \quad \tau=\frac{2 a}{V}, \\
& x=a \quad \Rightarrow \quad \tau=0 .
\end{aligned}
$$

Performing the integration in closed form on those terms where it is possible, the forces and aligning torques caused by tyre deformation can be expressed for the linear case as a function of the past and present values of the wheel centre point coordinate $Y_{\mathrm{T}}$ and the wheel angle $\psi$ for each wheel as:

$$
F=-2 a k Y_{\mathrm{T}}(t)-2 a d\left(\dot{Y}_{\mathrm{T}}(t)-V \psi(t)\right)+k V \int_{0}^{\frac{2 a}{V}} Y_{\mathrm{T}}(t-\tau)+a \psi(t-\tau) \mathrm{d} \tau
$$

and

$$
M=-\frac{2}{3} a^{3} k \psi(t)-\frac{2}{3} a^{3} d \dot{\psi}(t)+k V \int_{0}^{\frac{2 a}{V}}(a-V \tau)\left(Y_{\mathrm{T}}(t-\tau)+a \psi(t-\tau)\right) \mathrm{d} \tau .
$$

\section{Governing equations of the car-trailer combination with delayed tyre model}

The linearised deformation function Eq. (27) of the tyre is based on the wheel centre point coordinates and on the wheel orientation angle. Hence, the position vectors of the wheel centre points $T_{1}, T_{2}$ and $T_{3}$ (see Fig. 1) have to be determined as a function of the state variables of the mechanical model:

$$
\begin{aligned}
& {\left[\begin{array}{c}
X_{\mathrm{T}_{1}} \\
Y_{\mathrm{T}_{1}}
\end{array}\right]=\left[\begin{array}{c}
X_{1}+f \cos \psi_{1} \\
Y_{1}+f \sin \psi_{1}
\end{array}\right], \quad\left[\begin{array}{c}
X_{\mathrm{T}_{2}} \\
Y_{\mathrm{T}_{2}}
\end{array}\right]=\left[\begin{array}{c}
X_{1}-b \cos \psi_{1} \\
Y_{1}-b \sin \psi_{1}
\end{array}\right],} \\
& {\left[\begin{array}{c}
X_{\mathrm{T}_{3}} \\
Y_{\mathrm{T}_{3}}
\end{array}\right]=\left[\begin{array}{c}
X_{1}-h \cos \psi_{1}-l \cos \psi_{2} \\
Y_{1}-h \sin \psi_{1}-l \sin \psi_{2}
\end{array}\right] .}
\end{aligned}
$$

If we evaluate the linearised form of these expressions in Eq. (37) and Eq. (38) for each tyre, we obtain the forces and aligning torques. Here we present the expressions for the case when the lengths of the contact patches and the distributed stiffness are the same for all the tyres. Of course, this simplification slightly limited the validity of our results since the tyre parameters are different in practice due to the different vertical load on the tyres. Moreover, the location of the payload on the trailer have relevant effect on these vertical loads and we do not take into account this effect of the payload position. However, the applied simplification enables us to transform some terms more manageable form in the equations. Thus, the tyre forces and aligning torques can be written as

$$
\begin{aligned}
F_{1}= & -2 a k\left(Y_{1}(t)+f \psi_{1}(t)\right)-2 a d\left(\dot{Y}_{1}(t)+f \dot{\psi}_{1}(t)-V \psi_{1}(t)\right) \\
& +k V \int_{0}^{\frac{2 a}{V}} Y_{1}(t-\tau)+(f+a) \psi_{1}(t-\tau) \mathrm{d} \tau, \\
F_{2}= & -2 a k\left(Y_{1}(t)-b \psi_{1}(t)\right)-2 a d\left(\dot{Y}_{1}(t)-b \dot{\psi}_{1}(t)-V \psi_{1}(t)\right) \\
& +k V \int_{0}^{\frac{2 a}{V}} Y_{1}(t-\tau)-(b-a) \psi_{1}(t-\tau) \mathrm{d} \tau, \\
F_{3}= & -2 a k\left(Y_{1}(t)-h \psi_{1}(t)-l \psi_{2}(t)\right)-2 a d\left(\dot{Y}_{1}(t)-h \dot{\psi}_{1}(t)-l \dot{\psi}_{2}(t)-V \psi_{2}(t)\right) \\
& +k V \int_{0}^{\frac{2 a}{V}} Y_{1}(t-\tau)-h \psi_{1}(t-\tau)-(l-a) \psi_{2}(t-\tau) \mathrm{d} \tau,
\end{aligned}
$$


and

$$
\begin{aligned}
M_{1}= & -\frac{2}{3} a^{3} k \psi_{1}(t)-\frac{2}{3} a^{3} d \dot{\psi}_{1}(t) \\
& +k V \int_{0}^{\frac{2 a}{V}}(a-V \tau)\left(Y_{1}(t-\tau)+(f+a) \psi_{1}(t-\tau)\right) \mathrm{d} \tau \\
M_{2}= & -\frac{2}{3} a^{3} k \psi_{1}(t)-\frac{2}{3} a^{3} d \dot{\psi}_{1}(t) \\
& +k V \int_{0}^{\frac{2 a}{V V}}(a-V \tau)\left(Y_{1}(t-\tau)-(b-a) \psi_{1}(t-\tau)\right) \mathrm{d} \tau \\
M_{3}= & -\frac{2}{3} a^{3} k \psi_{2}(t)-\frac{2}{3} a^{3} d \dot{\psi}_{2}(t) \\
& +k V \int_{0}^{\frac{2 a}{V}}(a-V \tau)\left(Y_{1}(t-\tau)-h \psi_{1}(t-\tau)-(l-a) \psi_{2}(t-\tau)\right) \mathrm{d} \tau .
\end{aligned}
$$

Transform the governing equations Eq. (12) into the space of the generalized coordinates. For small oscillations around the rectilinear motion, the relationship between the generalized coordinates and pseudo velocities simplifies to:

$$
\begin{aligned}
& \beta_{1}=\dot{Y}_{1}-V \psi_{1}, \\
& \beta_{2}=\dot{\psi}_{1}, \\
& \beta_{3}=\dot{\psi}_{2} .
\end{aligned}
$$

Taking these into account, the linearised form of the equations of motion Eq. (12) can be written as

$$
\begin{aligned}
& m_{1} \ddot{Y}_{1}+m_{2}\left(\ddot{Y}_{1}-h \ddot{\psi}_{1}-l_{c} \ddot{\psi}_{2}\right)=F_{1}+F_{2}+F_{3}, \\
& J_{\mathrm{C}_{1}} \ddot{\psi}_{1}+m_{2}\left(h^{2} \ddot{\psi}_{1}-h \ddot{Y}_{1}+h l_{c} \ddot{\psi}_{2}\right)=F_{1} f-F_{2} b-F_{3} h+M_{1}+M_{2}, \\
& J_{\mathrm{C}_{2}} \ddot{\psi}_{2}+m_{2}\left(f^{2} \ddot{\psi}_{1}-f \ddot{Y}_{1}+h l_{c} \ddot{\psi}_{2}\right)=M_{3}-F_{3} l .
\end{aligned}
$$

After substitution of the formulas Eq. (40) and Eq. (41) into Eq. (43) we obtain a system of DDEs with distributed time delay:

$$
\mathbf{M} \ddot{\mathbf{y}}(t)+\mathbf{D} \dot{\mathbf{y}}(t)+\mathbf{K y}(t)=\mathbf{Q}\left(\mathbf{y}_{t}\right),
$$

where $\mathbf{y}(t)$ is the vector of the remaining state variables of the linearised system:

$$
\mathbf{y}(t)=\left[\begin{array}{l}
Y_{1}(t) \\
\psi_{1}(t) \\
\psi_{2}(t)
\end{array}\right],
$$

and $\mathbf{y}_{t}$ is a function of bounded variation above the delay interval $[0,2 a / V]$ defined by the shift operator $\mathbf{y}_{t}(\tau)=\mathbf{y}(t-\tau)$, $\tau \in[0,2 a / V]$. The mass matrix $\mathbf{M}$ can be expressed as

$$
\mathbf{M}=\left[\begin{array}{ccc}
m_{1}+m_{2} & -m_{2} h & -m_{2} l_{\mathrm{c}} \\
-m_{2} h & J_{\mathrm{C}_{1}}+m_{2} h^{2} & m_{2} h l_{\mathrm{c}} \\
-m_{2} l_{\mathrm{c}} & m_{2} h l_{\mathrm{c}} & J_{\mathrm{C}_{2}}+m_{2} l_{\mathrm{c}}{ }^{2}
\end{array}\right] .
$$

The stiffness matrix $\mathbf{K}$ can be separated into symmetric and non-symmetric parts, i.e., $\mathbf{K}=\mathbf{K}_{k}+\mathbf{K}_{d}$. The symmetric part is originated in the elastic properties of the tyres while the non-symmetrical part is originated in the tyre damping:

$$
\mathbf{K}_{k}=2 a k\left[\begin{array}{ccc}
3 & f-b-h & -l \\
f-b-h & f^{2}+b^{2}+h^{2}+\frac{2}{3} a^{2} & l h \\
-l & l h & l^{2}+\frac{1}{3} a^{2}
\end{array}\right],
$$




$$
\mathbf{K}_{d}=2 a d V\left[\begin{array}{ccc}
0 & -2 & -1 \\
0 & -f+b & h \\
0 & 0 & l
\end{array}\right] .
$$

The damping matrix $\mathbf{D}$ is proportional to the symmetric part of the stiffness matrix:

$$
\mathbf{D}=\frac{d}{k} \mathbf{K}_{k}
$$

The retarded functional $\mathbf{Q}\left(\mathbf{y}_{t}\right)$ on the right hand side of Eq. (44) can be composed as:

$$
\mathbf{Q}\left(\mathbf{y}_{t}\right)=k V \int_{0}^{\frac{2 a}{V}}\left(\mathbf{B}_{0}-V \tau \mathbf{B}_{1}\right) \mathbf{y}(t-\tau) \mathrm{d} \tau
$$

where the coefficient matrices are:

$$
\mathbf{B}_{0}=\left[\begin{array}{ccc}
3 & f-b-h+2 a & -l+a \\
f-b-h+2 a & f^{2}+b^{2}-h^{2}+2 a(f-b+a) & h(l-a) \\
-l+a & h(l-a) & (l-a)^{2}
\end{array}\right]
$$

and

$$
\mathbf{B}_{1}=\left[\begin{array}{ccc}
0 & 0 & 0 \\
2 & a-b+2 a & 0 \\
1 & -h & -l+a
\end{array}\right]
$$

\section{Linear stability and self-excited vibrations}

The characteristic function of the system can be determined by using the trial solution $\mathbf{y}(t)=\mathbf{A} \mathrm{e}^{\lambda t}$, where $\lambda \in \mathbb{C}$ is the characteristic exponent, $\mathbf{A} \in \mathbb{C}^{3}$. Substituting this into the differential equation Eq. (41) we obtain:

$$
\left(\lambda^{2} \mathbf{M}+\lambda \mathbf{D}+\mathbf{K}-k V\left(\int_{0}^{\frac{2 a}{V}} \mathbf{B}_{0} \mathrm{e}^{-\lambda \tau} \mathrm{d} \tau-\int_{0}^{\frac{2 a}{V}} V \tau \mathbf{B}_{1} \mathrm{e}^{-\lambda \tau} \mathrm{d} \tau\right)\right) \mathbf{A} \mathrm{e}^{\lambda t}=0,
$$

where the closed form calculation of the integrals leads to the characteristic equation

$$
\begin{aligned}
D(\lambda):= & \operatorname{det}\left(\lambda^{2} \mathbf{M}+\lambda \mathbf{D}+\mathbf{K}-k \frac{V}{\lambda}\left(\mathbf{B}_{0}\left(1-\mathrm{e}^{-\frac{2 a}{V} \lambda}\right)\right)\right. \\
& \left.-\mathbf{B}_{1}\left(\frac{V}{\lambda}\left(1-\mathrm{e}^{-\frac{2 a}{V} \lambda}\right)-2 a \mathrm{e}^{-\frac{2 a}{v} \lambda}\right)\right)=0,
\end{aligned}
$$

with $D(\lambda)$ defined as the characteristic function of the system.

It can be shown, that this equation has at least two zero roots, which is corroborated by our physical view, namely, the stability of the rectilinear motion is independent to the direction and position in the $(X, Y)$ reference coordinate plane. Therefore, from the point of view of the stability it is sufficient to analyse the simplified $\hat{D}(\lambda)=D(\lambda) / \lambda^{2}$ characteristic function.

The rectilinear motion of the vehicle is stable if and only if all the (infinitely many) characteristic roots take place on the left half of the complex plane, i.e.

$$
\operatorname{Re}\left(\lambda_{j}\right)<0, \quad j=1, \ldots, \infty .
$$

We investigate the stability of the rectilinear motion of the vehicle with special attention to the location of the payload on the trailer and to the longitudinal speed $V$ of the vehicle. Thus, we introduce the dimensionless payload position parameter

$$
p=\frac{l_{\mathrm{c}}}{l},
$$




\begin{tabular}{lcc}
\hline Parameter & Notation & Value \\
\hline Mass of the car & $m_{1}$ & $1473 \mathrm{~kg}$ \\
Mass of the trailer & $m_{2}$ & $879 \mathrm{~kg}$ \\
Yaw inertia of the car & $J_{\mathrm{C}_{1}}$ & $2500 \mathrm{kgm}^{2}$ \\
Yaw inertia of the trailer & $J_{\mathrm{C}_{2}}$ & $2601 \mathrm{kgm}^{2}$ \\
The distance between the car's front axle and the centre of gravity & $f$ & $1.1 \mathrm{~m}$ \\
The distance between the car's rear axle and the centre of gravity & $b$ & $1.6 \mathrm{~m}$ \\
The distance between the king pin and the centre of gravity of the car & $h$ & $2.7 \mathrm{~m}$ \\
The distance between the king pin and axle of the trailer & $l$ & $3.8 \mathrm{~m}$ \\
Half length of the tyre-ground contact patch & $a$ & $0.05 \mathrm{~m}$ \\
Distributed lateral stiffness of the tyre & $k$ & $1.2 \times 10^{7} \mathrm{~N} / \mathrm{m}^{2}$ \\
\hline
\end{tabular}

Table 1: The realistic parameter set-up used for the stability analysis.

which describes the location of the centre of gravity of the trailer with respect to the location of the trailers axle (e.g. $p=0$ if the centre of gravity is exactly at the king pin, and $p=1$ if the centre of gravity is exactly at the axle of the trailer.)

In view of the loss of stability, two cases can be differentiated. When a pure real characteristic root becomes positive, we can speak about 'static' loss of stability, which corresponds to saddle-node (SN) bifurcation of the steady state solution of the corresponding non-linear system. Then at the stability boundary the following equation has to be satisfied:

$$
\lim _{\lambda \rightarrow 0} \hat{D}(\lambda)=0,
$$

from which the critical parameter $p_{\mathrm{SN}}$ can be calculated in closed form. Here we present the formula for the undamped $(d=0)$ case:

$$
p_{\mathrm{SN}}=p_{0}-p_{-2} \frac{1}{V^{2}},
$$

where

$$
\begin{aligned}
p_{0} & =\frac{(3 l+a)\left(\left(m_{1}+m_{2}\right)(3(f-b)-2 a)+6 m_{2} h\right)}{3(3(f-b+2 h)-2 a) m_{2} l}, \\
p_{-2} & =\frac{2 a^{2} k\left(3(f+b)^{2}(a+3 l)-a^{2}(f+2(-a+h-2 l)-b)\right)}{3(3(f-b+2 h)-2 a) m_{2} l} .
\end{aligned}
$$

For realistic system parameters $p_{-2}>0$, which means that for $p<p_{0}$ there always exists a critical speed over which the rectilinear motion of the vehicle is unstable.

When the real part of a complex conjugate characteristic-pair becomes positive, the rectilinear motion loses its stability via oscillation (i.e. Hopf bifurcation may occur). For this case:

$$
\left.\hat{D}(\lambda)\right|_{\lambda= \pm \mathrm{i} \omega}=0 .
$$

Since this equation is transcendental, numerical methods have to be applied in order to detect the stability boundaries, like the so-called multi-dimensional bisection method [19]. Still, in certain undamped cases, semi-analytical solutions also help to check the numerical results, just like in [16]. There are several methods in the literature to select stable parameter domains from the many determined by the calculated boundaries [20]. Or one can use the semi-discretisation method (see [21]) to detect the stable parameter domain of linear DDEs.

Using these methods, stability charts were constructed in the $V-p$ parameter plane using the system parameters of Table 1. The parameters are based on the experimental study of Darling et al. [22], in which some of the parameters $\left(f, b, h, J_{\mathrm{C}_{1}}, a\right.$, and $\left.k\right)$ are not directly given. Consequently, they are chosen using the additional specifications of the experimental set-up in [22].

The stability analysis partly provided results with good agreement with former studies, namely, two unstable domains occur at large longitudinal velocities (see the shaded domains in Fig 3). One of them corresponds to the SN 


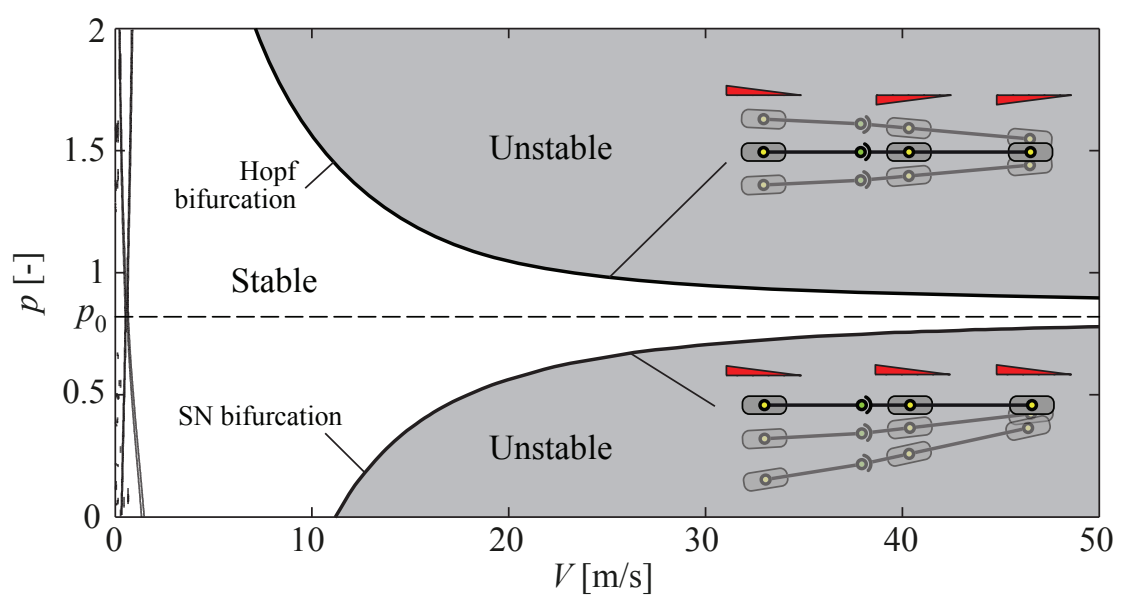

Figure 3: Linear stability chart for a wide speed range of the vehicle in the plane of the longitudinal speed $V$ and payload position parameter $p$. Tyre damping is not considered $(d=0)$. Unstable domains are shaded. Vibration modes with the typical tyre deformation shapes are also shown.

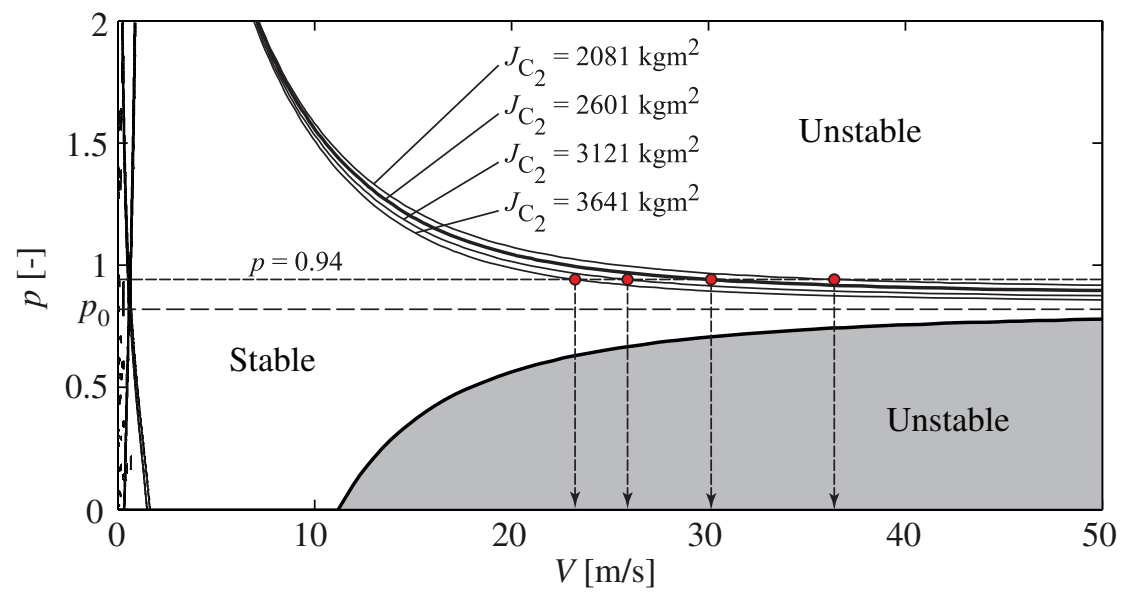

Figure 4: Stability boundaries constructed for different yaw inertias of the trailer. The system parameters are based on the experiments in [22]. The critical speeds are marked for $p=0.94$.

bifurcation curve given by Eq. (58). For the chosen parameters, this stability boundary can clearly be identified for $p<p_{0}=0.82$. The stability boundary for $p>p_{0}$ belongs to Hopf bifurcation, i.e. self-excited vibrations appear when this boundary is crossed. By means of the stability chart, it can be established that $p \approx p_{0}$ can guaranty the linear stability for a wide range of the speed. This statement is well-known in vehicle dynamics, and such condition can be proven even for a simple towed rigid-wheel with single contact point [17].

It is worth comparing these theoretical stability boundaries to the experimental results of [22]. Darling et al. analyse the effect of the different parameters of the caravan, but now we focus only on the effect of the yaw inertia $J_{\mathrm{C}_{2}}$ of the trailer. The stability boundaries are constructed for the different values of the yaw inertia in Fig. 4 . The experimental set-up considers $p=0.94$, which leads to the critical speeds given in Table 2, where the experimental results of Darling et al. are also presented.

In Fig 3, the emerging vibrations are also illustrated with the characteristic tyre deformation shapes for two essential types of the unstable domains. Note that the lateral tyre deformations seem to be close to simple linear functions, which clearly shows why the quasi-static tyre models (like Pacejka's creep-force model) can efficiently be used to identify these relevant stability boundaries, why these unstable domains were already verified in many publications (see for example, [12, 14, 22]).

In Fig. 5, simulation results are presented for a small impact-like perturbation of the trailer in the linearly stable 


\begin{tabular}{cccr}
\hline \multicolumn{4}{c}{ Critical speed [m/s] } \\
$J_{\mathrm{C}_{2}}\left[\mathrm{kgm}^{2}\right]$ & Calculated & Measured in [22] & Relative error \% \\
\hline 2081 & 36.9 & 33.5 & 10.1 \\
2601 & 30.2 & 29.1 & 3.8 \\
3121 & 26.3 & 27.3 & -3.7 \\
3641 & 23.5 & 25.5 & -7.8 \\
\hline
\end{tabular}

Table 2: Comparison of the theoretical and the measured critical speeds.
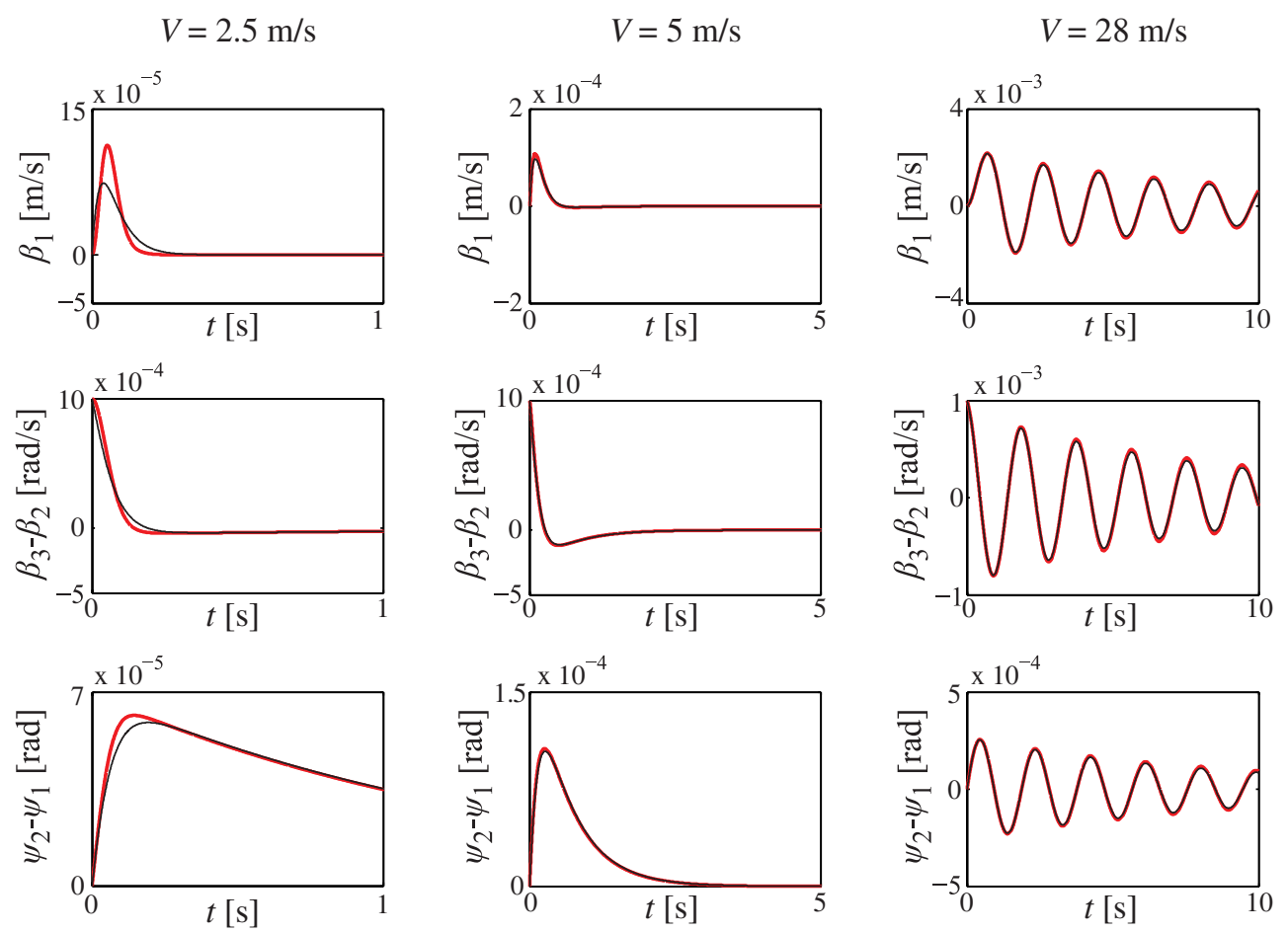

Figure 5: Small amplitude vibrations of the car-trailer combination simulated with the time delayed (red) and with the steady-state (black) tyre models for different longitudinal speeds $V$.

parameter domain of Table 1 when $p=0.94$ is used. Time histories are determined for the longitudinal speeds $2.5,5$ and $28 \mathrm{~m} / \mathrm{s}$ (see the stable domain in Fig.4) using both the time delayed tyre model (red curves) and the creep-force model (black curves). As it can be observed, for large speeds both tyre models provide very similar results while at low speeds relatively large discrepancies can be identified. The memory effect is relevant at low speed, but the implementation of the time delay model can be beneficial if numerical simulations are carried out for (occasionally quickly) varying speeds since this tyre model does not have any limitation for the applicable speed range. It works with limitation for turn-slip [23] since it does not describe the self-aligning moment generated by the different longitudinal slips along the width of the contact patch. Moreover, the delayed tyre model does not involve the sliding effect in its present form, which limits its usability in the non-linear domain.

In this study, new unstable parameter domains of the car-trailer combination are also discovered for small speeds, which can be only determined by taking into account the regenerative effect of the tyre-ground contact patch. Fig. 6 shows the charted stability boundaries, which all relates to Hopf bifurcations leading to the oscillation of the vehicle. If the speed tends to zero $(V \rightarrow 0)$, an infinite number of such unstable domains emerge, which is typical in delayed systems (e.g. see 'chatter' of machine tools in [21] or 'wheel shimmy' in [16]). Note that the vibration frequency $\omega$ also tends to infinity as the speed decreases, which means that the wave length of the tyre deformation shape also decreases. Fig. 6 presents some of these unstable domains. 


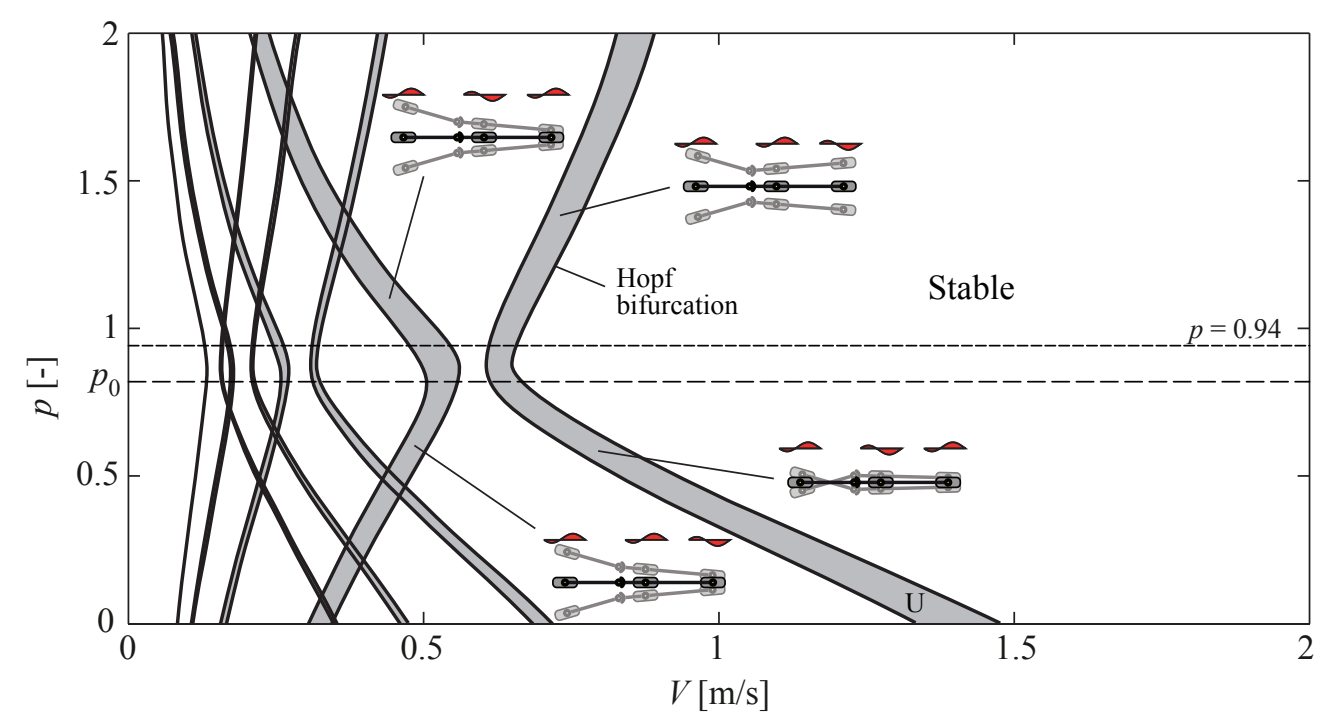

Figure 6: Stability chart for the slow motion of the vehicle in the plane of the longitudinal speed $V$ and the payload position parameter $p$. Tyre damping is not considered $(d=0)$. The characterizing vibration modes and tyre deformations are shown for some of the unstable domains.

There are also parameter domains, where two unstable regions intersect each other. In these cases two pairs of complex conjugate roots of the characteristic equation have positive real parts, therefore, oscillations occur with multiple frequencies. For the case of the wheel shimmy, this type of intersections of stability boundaries was experimentally validated in [17] giving explanation for the quasi-periodic oscillation of a one degree-of-freedom caster-wheel system.

The detected stability boundaries can be characterized by the mode shape of the emerging vibration. This is demonstrated in Fig. 6. The deformed shapes of the tyres are all sinusoidal with period one in the investigated domains. The boundary condition of the brush tyre model ensures the zero deformation at the leading edge of the contact patch only, but one can observe zero or close to zero deformation at the trailing edge, too. Note, that the phase of the tyre deformations can be different in the simply connected unstable domain according to the payload location.

The effect of the tyre damping is analysed in Fig. 7, where the distributed damping parameter $d$ is increased with discrete steps. As it can be seen, the viscous damping reduces the size of the unstable regions, and the smaller very narrow unstable regions even disappear. Note that the rectilinear motion can be stabilized for a wide speed range by means of large enough damping values if $p=p_{0}$.

It is also interesting to investigate practical cases when the tyres of the vehicle have different parameters. Here, we vary the contact patch length $a_{3}$ and the stiffness $k_{3}$ of the trailer's tyre, which corresponds to the case when the trailer's tyres have smaller inflation pressure and/or larger vertical load. Experimental observations suggest that such conditions make the vehicle to be more 'unstable'. Fig. 8 shows the stability charts of the undamped $(d=0)$ system for different values of the contact patch length and stiffness. The stability chart in the right bottom panel corresponds to the reference system with data in Table 1. As the tyre stiffness is reduced, the unstable domains shrink and shift to the left. The effect of the variation of the contact patch length is more complicated. If the differences between the contact patch lengths are small, many unstable domains disappear; compare the cases $a_{3}=0.05 \mathrm{~m}$ and $a_{3}=0.0625 \mathrm{~m}$. However, when the tyre contact length $a_{3}$ of the trailer is $3 / 2$ or 2 times larger than the contact length of the other tyres, then some of the unstable domains rebirth and extend. This effect can be originated in the interactions of the tyres, namely, the stability depends on whether the tyres can excite each other or not, which corresponds to the wave lengths of the tyre deformations (check them in Fig. 6). All this means that there exist certain (maybe 'extreme') parameter set-ups, which can lead to kind of worst case scenarios.

\section{Conclusions}

Complex structures of unstable parameter domains were identified using the delayed tyre model in the single track mechanical model of the car-trailer combination. The intricate effects of the system parameters - with special 

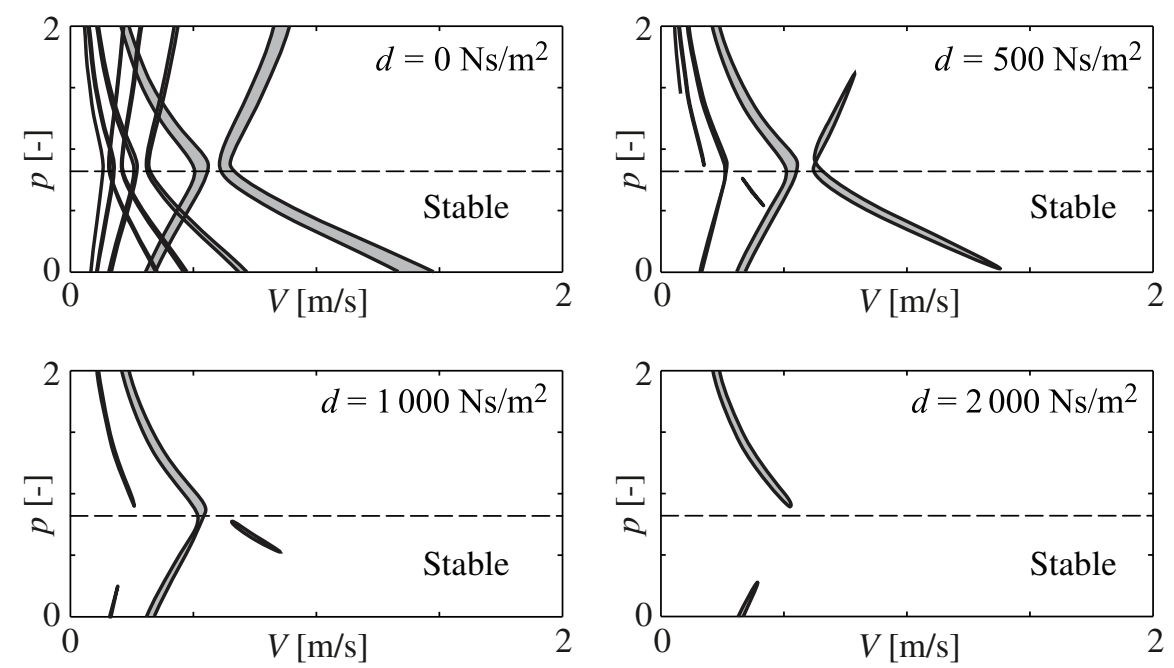

Figure 7: The effect of tyre damping on stability. Stability charts are constructed for different damping factors in the plane of the longitudinal speed $V$ and the payload position parameter $p$. Shaded domains are unstable.

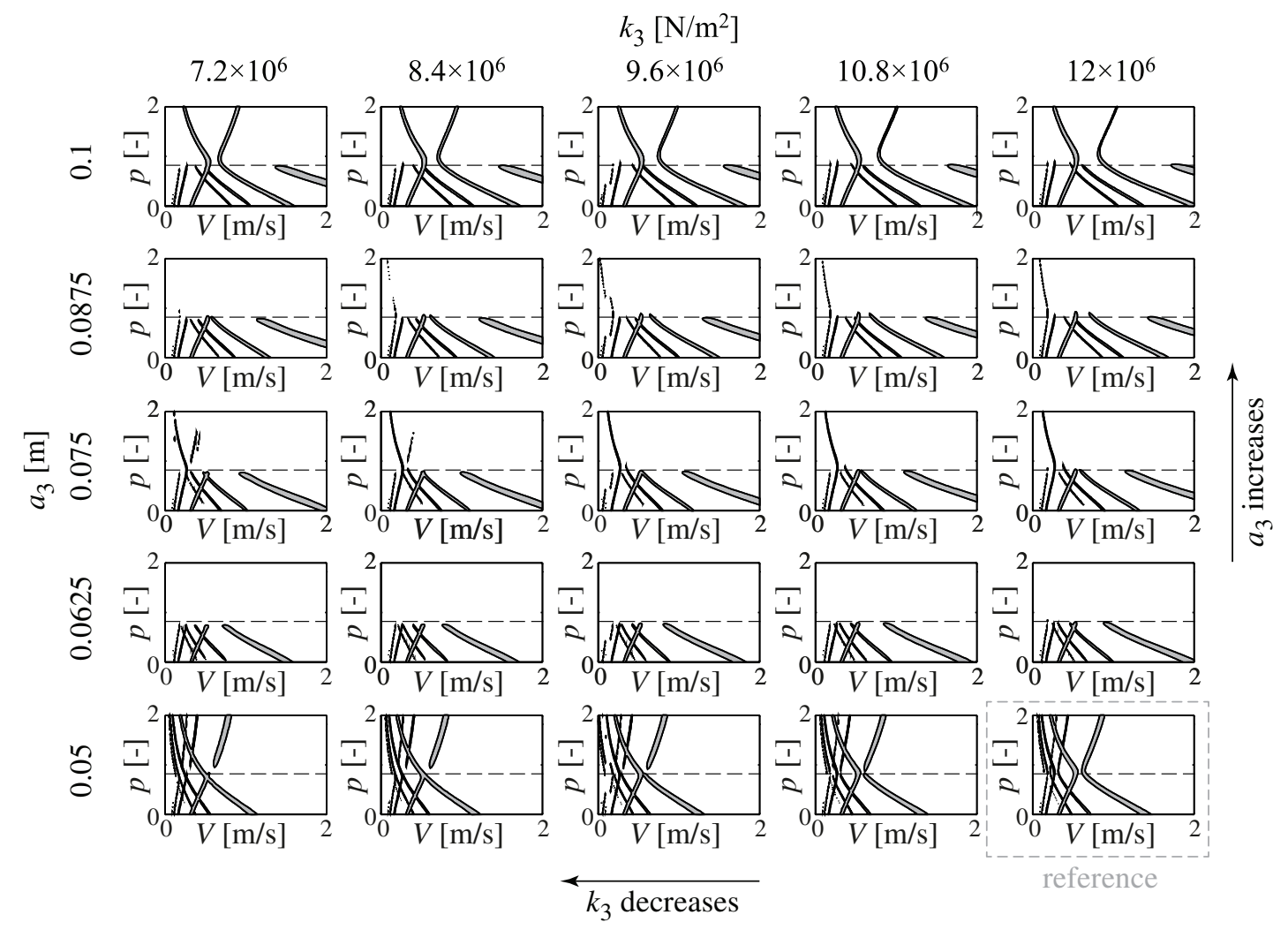

Figure 8: The effect of the contact patch length $a_{3}$ and the lateral stiffness $k_{3}$ of the trailer's tyre on the stability of the car-trailer system. Unstable domains are shaded in the plane of the longitudinal speed $V$ and the payload position parameter $p$. Tyre damping is considered to be zero $(d=0)$.

attention to the tyre specific stiffness, damping and the length of the contact patch - were analysed via the variation of the corresponding stability charts constructed in the plane of the longitudinal vehicle speed and the payload position parameter along the trailer. 
The numerical evaluations of the analytical results proved that although the emerging vibrations in the newly discovered unstable domains are 'weak' compared to the ones at the well-known unstable parameter domains at large longitudinal speeds. Due to non-linear effects like tyre-slip, the vibration amplitudes at these domains tend to be very small. Still, as shown in [10] for a shimmying tyre, the partial sliding of the contact patch induces enhanced heat generation causing higher fuel consumption and noise even when the vibrations are tiny.

Moreover, these instabilities may have a significant role in specific applications where accurate modelling of the tyre deformation is needed for the control of low-speed manoeuvres like parking with car-trailer systems or abrupt changes in speed and path-curvature. Also, the analytically detected unstable parameter ranges can be useful for the verification of multi-body simulation software in case of more complicated real-world car-trailer mechanical models.

\section{Acknowledgement}

This research was partly supported by the János Bolyai Research Scholarship of the Hungarian Academy of Sciences and by the Hungarian National Science Foundation under grant no. OTKA PD105442.

\section{References}

[1] H. B. Pacejka, The wheel shimmy phenomenon, Ph.D. thesis, Technical University of Delft, The Netherlands (1966)

[2] E. Coetzee, B. Krauskopf, M. H. Lowenberg, Nonlinear analysis of aircraft ground handling, in: Proceedings of ICGF $2006,2006$.

[3] C. Howcroft, B. Krauskopf, M. Lowenberg, S. Neild, Effects of freeplay on aircraft main landing gear stability, in: Proceedings of AIAA 2012, AIAA, Minneapolis, Minnesote, USA, 2012, pp. 1-16, paper No. AIAA 2012-4730.

[4] N. Terkovics, S. Neild, M. Lowenberg, B. Krauskpof, Bifurcation analysis of a coupled nose landing gear-fuselage system, in: Proceedings of AIAA 2012, AIAA, Minneapolis, Minnesote, USA, 2012, pp. 1-14, paper No. AIAA 2012-4731.

[5] P. Lugner, H. B. Pacejka, M. Plöchl, Recent advances in tyre models and testing procedures, Vehicle System Dynamics 43 (6) (2005) $413-426$.

[6] P. B. U. Andersson, W. Kropp, Rapid tyre/road separation: An experimental study of adherence forces and noise generation, Wear 266 (1-2) (2009) 129-138.

[7] D. O'Boy, A. Dowling, Tyre/road interaction noise - $\varnothing$ Numerical noise prediction of a patterned tyre on a rough road surface, Journal of Sound and Vibration 323 (1ф2) (2009) 270 - 291. doi:http://dx.doi.org/10.1016/j.jsv.2008.12.024.

[8] J. Cesbron, F. Anfosso-Lédée, D. Duhamel, H. P. Yin, D. L. Houédec, Experimental study of tyre/road contact forces in rolling conditions for noise prediction, Journal of Sound and Vibration 320 (1ф2) (2009) 125 - 144. doi:http://dx.doi.org/10.1016/j.jsv.2008.07.018.

[9] R. J. Pinnington, Tyre $\not$-road contact using a particle $\not$-envelope surface model, Journal of Sound and Vibration 332 (26) (2013) 7055 - 7075. doi:http://dx.doi.org/10.1016/j.jsv.2013.08.018.

[10] D. Takács, G. Stépán, Micro-shimmy of towed structures in experimentally uncharted unstable parameter domain, Vehicle System Dynamics 50 (11) (2012) 1613-1630. doi:10.1080/00423114.2012.691522.

[11] F. D. Rossa, G. Mastinu, C. Piccardi, Bifurcation analysis of an automobile model negotiating a curve, Vehicle System Dynamics 50 (10) (2012) 1539-1562. arXiv:http://dx.doi.org/10.1080/00423114.2012.679621, doi:10.1080/00423114.2012.679621. URL http://dx.doi.org/10.1080/00423114.2012.679621

[12] H. B. Pacejka, Tyre and Vehicle Dynamics, Elsevier Butterworth-Heinemann, Linacre House, Jordan Hill, Oxford OX2 8DP, 200 Wheeler Road, Burlington MA 01803, 2002.

[13] R. S. Sharp, M. A. A. Fernańdez, Car-caravan snaking - part 1: the influence of pintle pin friction, in: Proceedings of the Institution of Mechanical Engineers Part C - Journal of Mechanical Engineering Science, Vol. 216 of 7, 2002, pp. 707-722.

[14] H. Troger, K. Zeman, A nonlinear-analysis of the generic types of loss of stability of the steady-state motion of a tractor-semitrailer, Vehicle System Dynamics 13 (4) (1984) 161-172.

[15] D. Takács, G. Stépán, Contact patch memory of tyres leading to lateral vibrations of four-wheeled vehicles, Philosophical Transactions of the Royal Society A: Mathematical, Physical and Engineering Sciences 371 (1993). doi:10.1098/rsta.2012.0427.

[16] D. Takács, G. Orosz, G. Stépán, Delay effects in shimmy dynamics of wheels with stretched string-like tyres, European Journal of Mechanics A/Solids 28 (2009) 516-525.

[17] D. Takács, Dynamics of towed wheels - Nonlinear theory and experiments, Ph.D. thesis, Budapest University of Technology and Economics, Hungary (2010).

[18] F. Gantmacher, Lectures in analytical mechanics, MIR Publishers, Moscow, 1975.

[19] D. Bachrathy, G. Stépán, Bisection method in higher dimensions and the efficiency number, Periodica Polytechnica 56 (2) (2012) $81-86$.

[20] G. Stépán, Retarded Dynamical Systems, Longman Scientific and Technical, London, 1989.

[21] T. Insperger, G. Stépán, Semi-Discretization for Time-Delay Systems, Springer, New York, 2011.

[22] J. Darling, D. Tilley, B. Gao, An experimental investigation of car-trailer high-speed stability, Proceedings of the Institution of Mechanical Engineers, Part D: Journal of Automobile Engineering 223 (4) (2009) 471-484. doi:10.1243/09544070jauto981.

[23] H. B. Pacejka, Spin: camber and turning, Vehicle System Dynamics 43 (1) (2015) 3 - 17. 